\title{
Patients with chronic autoimmune thyroiditis are not at higher risk for developing clinically overt thyroid cancer: a 10-year follow-up study
}

\author{
Mario Rotondii ${ }^{1,2}$, Gloria Groppelli ${ }^{3}$, Laura Croce ${ }^{1,2,4}$, Francesco Latrofa $\mathbb{1}^{5}$, Giuseppe Ancona ${ }^{2}$, \\ Francesca Coperchini ${ }^{1}$, Daniela Pasquali ${ }^{6}$, Carlo Cappelli ${ }^{7}$, Alessandro Fugazza ${ }^{8}$, Valeria Guazzoni ${ }^{3}$, \\ Giorgio Radetti ${ }^{9}$ and Luca Chiovato ${ }^{1,2}$
}

${ }^{1}$ Istituti Clinici Scientifici Maugeri IRCCS, Unit of Internal Medicine and Endocrinology, Laboratory for Endocrine Disruptors, Pavia, Italy, ${ }^{2}$ Department of Internal Medicine and Therapeutics, University of Pavia, Pavia, Italy, ${ }^{3}$ Unit of Internal Medicine, Medical-Oncologic Department, ASST Lodi, Lodi, Italy, ${ }^{4}$ PhD course in Experimental Medicine, University of Pavia, Pavia, Italy, ${ }^{5}$ Department of Clinical and Experimental Medicine, University of Pisa, Pisa, Italy, ${ }^{6}$ Department Advanced Medical and Surgical Science, A.O.U. Vanvitelli, University of Campania 'Luigi Vanvitelli', Naples, Italy, ${ }^{7}$ Department of Clinical and Experimental Sciences, University of Brescia, Brescia, Italy, ${ }^{8}$ Digestive Endoscopy Unit, Department of Gastroenterology, Humanitas Research Hospital, Rozzano, Italy, and ${ }^{9}$ Marienklinik, Bolzano, Italy

Correspondence should be addressed to L Chiovato Email luca.chiovato@icsmaugeri.it

\begin{abstract}
Objective: The association between chronic autoimmune thyroiditis (CAT) and differentiated thyroid cancer (DTC) remains controversial. The incidence of DTC increases when screening procedures are implemented, as typically occurs in CAT patients being routinely submitted to thyroid ultrasound (US). The aim of this study was to longitudinally evaluate the long-term development of DTC in patients with CAT.

Design and methods: A retrospective longitudinal cohort study was designed. For the study, 510 patients with chronic autoimmune thyroiditis (CAT) with a 10-year follow-up were enrolled. Patients were divided in two groups according to the presence (CAT+ NOD+; $n=115$ ) or absence (CAT+ NOD-; $n=395$ ) of co-existent nodules at diagnosis. The main outcome measures were appearance of new thyroid-nodules and development of DTC during follow-up.

Results: During a 10-year median follow-up period, new thyroid-nodules were detected in 34/115 (29.5\%) patients in the CAT+ NOD+ group and in 41/395 (10.3\%) in the CAT+ NOD- group $(P<0.001)$. Logistic regression analysis showed that thyroid-volume at diagnosis and belonging to the CAT+ NOD+ group significantly predicted the appearance of a new thyroid nodule during follow-up, independently of baseline age and sex. Among the 75 patients experiencing the appearance of a new nodule, 27 (39\%) met the criteria for fine-needle-aspiration-cytology (FNAC). A benign cytological diagnosis was rendered in all cases.

Conclusions: In our series of CAT patients, the appearance of new thyroid-nodules was frequent, but none of them were found to be malignant. The presence of CAT appears to be associated with a negligible risk of developing clinically overt DTC.
\end{abstract}

European Journal of Endocrinology

(2020) 183, 317-323
Published by Bioscientifica Ltd. 


\section{Introduction}

The association between chronic autoimmune thyroiditis (CAT) and differentiated thyroid cancer (DTC), first proposed by Dailey et al. in 1955, is a widely debated issue (1). DTCs are frequently diagnosed in the context of CAT and, at histology, display an inflammatory-immune cell infiltration $(2,3)$. However, it is still a matter of debate whether chronic autoimmune inflammation is responsible for the malignant transformation or cancerspecific inflammatory molecules induce the peri-tumoral immune inflammation. The link between different types of cancer and inflammation has been recognized since more than a century $(4,5)$, but data regarding thyroid tumors are still controversial. Lots of studies investigated this association, but different approaches have led to discordant findings. A clear association between HT and differentiated thyroid cancer (DTC), mainly papillary, was found in surgical series $(6,7,8,9,10,11,12,13,14)$, whereas such an association was not found in cytological studies $(15,16,17,18,19,20,21,22)$. However, it should be acknowledged that the former studies might carry the risk for selection biases. In addition, Jackson et al. recently reported that chronic lymphocytic thyroiditis should be considered a risk factor for incidental thyroid cancer (23).

In a recent review of the literature, which included 11 papers for a total study population of 22619 subjects, the authors concluded for an overall relative-risk (RR) of 1.36 for PTC in CAT patients (24). Again, discrepant data emerged when comparing cytological as opposed to surgical series. The average prevalence of PTC in CAT patients was $1.20 \%$ $(\mathrm{RR}=0.69)$ in cytological series $(16,17)$ and increased up to $27.56 \% \quad(\mathrm{RR}=1.59)$ in archival thyroidectomy series $(6,12,14)$. This discrepancy may be explained by the fact that, because CAT per se does not represent an indication for thyroid surgery, retrospective analyses of surgical series introduce a selection bias $(17,24)$. Indeed, CAT patients undergo thyroidectomy either for the uncommon occurrence of huge Hashimoto's goiters producing compression or, more frequently, for the detection of cytologically suspicious or malignant nodules. The fact that most PTC are slowly growing and asymptomatic malignancies, and that their population incidence increases when screening procedures are implemented, add further arguments favoring a selection bias in surgical series $(25,26)$. Indeed, patients with CAT are likely to undergo thyroid ultrasound (US) examination both at diagnosis and during the follow-up.

The aim of our study was to longitudinally assess the possible long-term occurrence of DTC in a cohort of CAT patients undergoing regular US follow-up.

\section{Patients and methods}

The outpatients' Data Base of the Unit of Endocrinology of ICS Maugeri (Pavia, Italy) was searched for patients who received a diagnosis of CAT between 1997 and 2014 . Pavia, located in Northern Italy, is an area of mild iodine deficiency. Inclusion criteria were: (1) a diagnosis of CAT, as assessed by positive tests for circulating thyroglobulin (TgAb) and/or thyroid peroxidase (TPOAb) antibodies, and/or a hypoechoic pattern of the gland at US; (2) the availability of a thyroid US at diagnosis; (3) a benign cytology in patients initially presenting an associated thyroid nodule (NOD); and (4) a minimal follow-up period of 5 years, with a yearly thyroid US scan performed by the same operator.

The final study cohort included 510 patients (476 females and 34 males). In detail, 297 (58.2\%) patients showed positive tests for both TgAb and TPOAb; 134 (26.3\%) patients had only TPO Ab; 44 (8.6\%) had only $\mathrm{Tg} \mathrm{Ab}$; and 35 (6.9\%) patients had negative tests for both $\mathrm{Ab}$ and based upon a diffuse hypoechoic pattern of the thyroid at ultrasound were diagnosed with serum negative autoimmune thyroiditis $(27,28)$. Their median (range) age was 49 (19-82 years).

Patients were further subdivided in two groups according to the presence or absence of co-existent nodule/s at diagnosis. Group 1 (CAT+, NOD+) and group 2 (CAT+, NOD-) included 115 and 395 patients, respectively.

The median (range) follow-up period was 10 (5-17) years. The development of new thyroid nodule/s and/ or significant modifications in pre-existent nodules were registered throughout the follow-up.

All patients signed an informed consent concerning the future use of their clinical-pathological data for research purposes. This study was approved by the Ethical Committee of ICS Maugeri, Pavia (Protocol N. 2403 CE).

\section{Thyroid ultrasound}

Thyroid US evaluation was performed using a real-time US device equipped with a linear transducer operating at $7.5 \mathrm{MHz}$. All thyroid US scans were performed by the same experienced operator. Thyroid nodules were examined for the following US features: nature (i.e. solid, cystic, or mixed); echogenicity (i.e. hyperechoic, isoechoic, or hypoechoic compared with normal thyroid parenchyma and with neck muscles); size and 
homogeneity (i.e. homogeneous or inhomogeneous); microcalcifications (hyperechoic spots of at least 2 $\mathrm{mm}$ without acoustic shadowing); regular or irregular margins; halo sign (i.e. presence or absence of an hypoechoic rim); and pattern of color-flow Doppler. The mentioned characteristics were summarized according to the EU-TIRADS classification system (29). Thyroid volume was estimated by the elliptical shape volume formula (length $\times$ width $\times$ depth $\times 0.479$ ). Lateral neck lymph nodes were routinely examined.

\section{Fine needle-aspiration cytology (FNAC)}

FNAC was performed in all newly developing nodules when they were larger than $1 \mathrm{~cm}$ in maximum diameter or, irrespective of their size, when they showed suspicious features at US. FNAC was performed under US guidance by a skilled endocrinologist using a 23-gauge needle attached to a 2.5-mL syringe. Cytological samples were classified into six classes: THY1 (nondiagnostic), THY2 (benign), THY3 (Atypia of undetermined significance or follicular lesion of undetermined significance), THY4 (follicular neoplasm or suspicious for a follicular neoplasm), THY5 (suspicious for malignancy), and THY6 (malignant), according to the Bethesda classification (30). None of the lymph node was found to require FNAC.

\section{Statistical analysis}

Statistical analysis was performed using the SPSS Software (SPSS, Inc.). Between-groups comparisons were performed using the Student's t-test for unpaired data and the Mann-Whitney $U$-test according to a normal or a non-parametric distribution. Within-group comparisons were performed using the Student's $t$-test for paired data and the Wilcoxon's test according to a normal or a nonparametric distribution. Frequencies among groups were compared using the $\chi^{2}$-test with Fisher's correction when appropriate. To test the effects of different variables independent of a covariate, multivariate logistic regression analysis was used and partial correlation coefficients were computed. The multivariate model was constructed by entering the occurrence of a new nodule as a dependent variable, while age, gender, thyroid volume at baseline and the Group (CAT+ NOD+ and CAT+ NOD-) were entered as covariates.

Kaplan-Meier estimates were used to generate an overall survival curve for new nodules occurrence and differences among CAT+ NOD+ and CAT+ NOD- groups were assessed by log-rank test. A $P$ value of $<0.05$ was considered statistically significant. Results are expressed as mean \pm s.D., unless otherwise stated.

\section{Results}

Table 1 shows demographic data and thyroid US features in $\mathrm{CAT}+\mathrm{NOD}+$ and in CAT+ NOD- groups. Patients in the two subgroups had a similar gender ratio, but those in the CAT+ NOD+ group were older and had a larger thyroid gland at diagnosis. The two groups were characterized by similar frequencies of patients displaying both $\mathrm{Tg} \mathrm{Ab}$ and TPO Ab, TPO Ab alone, Tg Ab alone and negative tests for $\mathrm{Tg} \mathrm{Ab}$ and TPO $\mathrm{Ab}$ in the presence of a diffuse hypoechoic pattern of the thyroid parenchyma at US. Compared to the CAT+ NOD+ group, the CAT+ NODone comprised a higher percentage of patients who were on LT4 replacement therapy when they entered the study or who were started on LT4 replacement therapy just at the beginning of the follow-up. In the CAT+ NOD- group

Table 1 Demographic data, thyroid function and thyroid autoantibody status and US features in the CAT+ NOD+ and CAT+ NOD-groups.

\begin{tabular}{|c|c|c|c|}
\hline & CAT+, NOD+ $(n=115)$ & CAT+, NOD- $(n=395)$ & $P$ value \\
\hline $\mathrm{M} / \mathrm{F}$ & $7 / 108$ & $27 / 368$ & 0.486 \\
\hline Age (years; median (range)) & $52(21-81)$ & $48(19-82)$ & $<0.001$ \\
\hline Thyroid volume (mL; median (range)) & $13(4-64)$ & $10(2-70)$ & $<0.001$ \\
\hline \multicolumn{4}{|l|}{ Serum TSH (median, 5th-95th centiles) } \\
\hline At study entry & $2.4(0.6-11.37)$ & $4.38(10.73-38.0)$ & $<0.001$ \\
\hline At last evaluation & $1.76(0.50-5.72)$ & $2.14(0.60-6.57)$ & $<0.05$ \\
\hline Thyroid antibody status & & & 0.586 \\
\hline $\operatorname{TgAb}+/ \operatorname{TPOAb+}$ & $62(53.9 \%)$ & $235(59.5 \%)$ & \\
\hline $\operatorname{TgAb}+/ \operatorname{TPOAb}-$ & $13(11.3 \%)$ & $31(7.8 \%)$ & \\
\hline $\operatorname{TgAb}-/ T P O A b+$ & $31(27.0 \%)$ & $103(26.1 \%)$ & \\
\hline TgAb-/TPOAb- & $9(7.9 \%)$ & $26(6.6 \%)$ & \\
\hline
\end{tabular}


143/395 (36.2\%) patients were receiving LT4 when they entered the study, the correspondent figure in the CAT+ NOD+ group was 29/115 (25.2\%); $P<0.05$. The median serum level of TSH at study entry was higher in the CAT+ NOD- as compared to CAT+ NOD+ (4.38 vs $2.14 \mu \mathrm{U} / \mathrm{mL}$; $P<0.001)$ group. At the last evaluation, the difference was less evident although still significant (2.4 vs $1.76 \mu \mathrm{U} / \mathrm{mL}$; $P<0.05)$. Similarly, $154 / 252(61.1 \%)$ patients in the CAT+ NOD- group and 31/86 (36.0\%) in the CAT+ NOD+ one $(P<0.001)$ were started on LT4 when recruited. The overall median and range follow-up time was 10 years $(5-17)$ in the whole cohort, without significant differences between the two subgroups (11 (5-16) years for CAT+ NOD+ vs 10 (5-17) years for CAT+ NOD-; $P=0.083$ ).

Throughout the follow-up period, the US appearance of new thyroid nodules was detected in 75 out of 510 (14.7\%) patients of the whole cohort. When patients' subgroups were analyzed separately, findings differed in that 34 out of 115 (29.5\%) patients in the CAT+ NOD+ subgroup developed new nodules as compared with a significantly lower frequency in the CAT+ NOD- subgroup: 41 out of $395(10.3 \%)$ patients $(P<0.001)$ (Fig. 1$)$. The median and range time for new nodules appearance was 4 (1-13) years. As shown in Fig. 2, life time survival analysis for new nodule development confirmed a significant difference between the two subgroups, with a higher risk for new nodule development in the CAT+ NOD+ subgroup (Kaplan-Meier Log Rank 24.441, $P<0.001$ ).

Twenty-seven out of $75(36 \%)$ patients experiencing the appearance of a new nodule met the US criteria for performing a FNAC. In 23 of them (85\% of cases), FNAC was performed due to the detection of a $>1 \mathrm{~cm}$ in maximum diameter nodule, whereas in four patients (15\%) the indication for FNAC derived from suspicious US features. No difference in the rate of nodules requiring

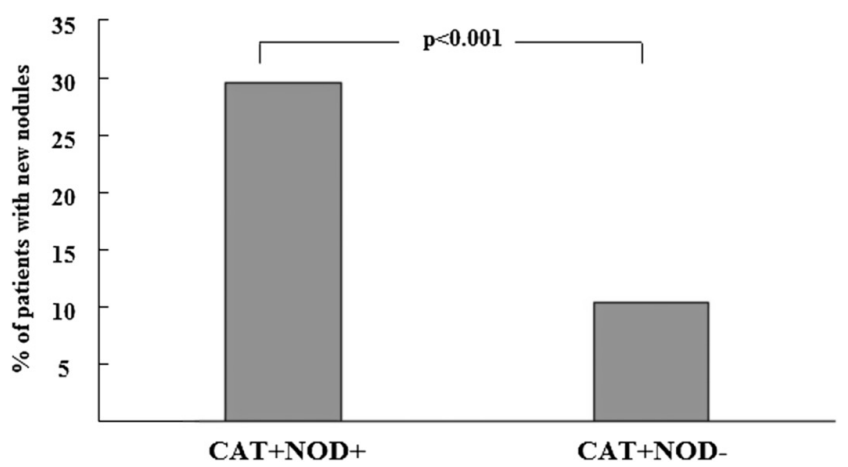

Figure 1

Percentage of patients who developed new nodules during the follow-up.
FNAC was observed in the two subgroups of patients (14 out of 34 patients in the CAT+ NOD+ and 13 out of 41 patients in the CAT+ NOD-; $P=0.472$ ). A benign cytology was observed in all the 27 patients who underwent FNAC (Table 2). The ultrasound characteristics at the end of the follow-up of both nodules requiring or not requiring FNAC are shown in Table 2. We also compared the median follow-up time calculated from the first ultrasound detection of a new nodule and the end of the follow-up in the two subgroups of nodules (6 (0-11) years vs $3.5(0-11)$ years, respectively; $P=0.051)$. Although statistical significance was not reached, nodules requiring FNAC showed a trend for a longer follow-up time. Table 1 summarizes the ultrasound features at the end of the follow-up of nodules requiring or not requiring FNAC.

The different rate of new nodule appearance between the two subgroups prompted further investigation. A multiple binary logistic regression model was constructed entering the appearance of new nodules (yes/no) as dependent variable and age, sex, thyroid volume at diagnosis, subgroup of patients (CAT+ NOD+/CAT+ NOD-), and number of nodules at first US as covariates. As shown in Table 3, multiple regression analysis confirmed that patients in the CAT+ NOD+ subgroup carried a significantly higher risk for developing new thyroid

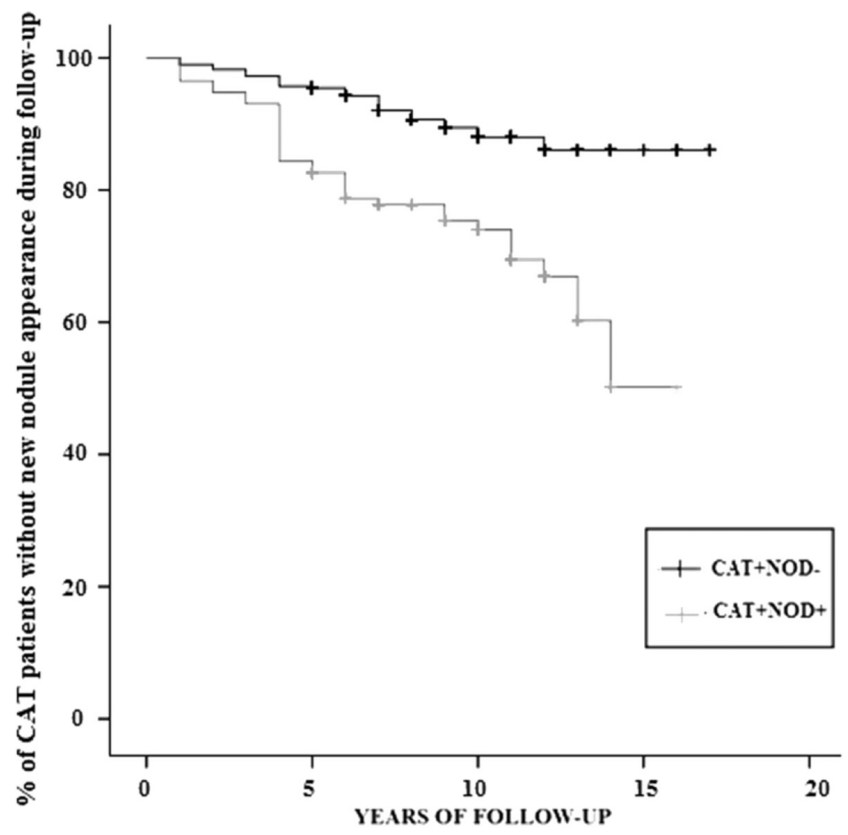

Figure 2

Survival analysis for the development of new nodules in the CAT+ NOD+ and CAT+ NOD- subgroups. (Kaplan-Meier Log Rank 24.441, $P<0.001)$. 
Table 2 Appearance of new nodules in CAT+ NOD+ and CAT+ NOD- groups and corresponding ultrasound and FNAC results.

\begin{tabular}{l}
\hline \\
\hline Follow-up time (years; (median and range)) \\
Patients developing neo nodules $(n(\%))$ \\
Nodules not requiring FNAC $(\%)$ \\
Maximum diameter ( $\mathrm{mm})$ \\
EUTIRADS \\
3 \\
4 \\
Nodules requiring FNAC $(\%)$ \\
Maximum diameter $(\mathrm{mm})$ \\
EUTIRADS \\
3 \\
4 \\
5 \\
Benign cytology
\end{tabular}

\begin{tabular}{c}
\hline CAT+, NOD+ $(n=115)$ \\
\hline $11(5-16)$ \\
$34 / 115(29.5 \%)$ \\
$20 / 34(58.8 \%)$ \\
$7.5 \pm 1.6$ \\
$13 / 20(65.0 \%)$ \\
$7 / 20(35.0 \%)$ \\
$14 / 34(41.2 \%)$ \\
$13.2 \pm 3.7$ \\
$9 / 14(64.3 \%)$ \\
$3 / 14(21.4 \%)$ \\
$2 / 14(14.3 \%)$ \\
$14 / 14(100 \%)$ \\
\hline
\end{tabular}

\begin{tabular}{ccr}
\hline CAT+, NOD $-(n=395)$ & & P value \\
$10(5-17)$ & & 0.083 \\
$41 / 395(10.3 \%)$ & & 0.001 \\
$28 / 41(68.3 \%)$ & & 0.472 \\
$6.6 \pm 2.0$ & 0.108 \\
& 0.297 \\
$22 / 28(78.6 \%)$ & \\
$6 / 28(21.4 \%)$ & \\
$13 / 41(31.7 \%)$ & 0.472 \\
$12.8 \pm 4.3$ & 0.831 \\
& \\
$8 / 13(51.5 \%)$ & \\
$3 / 13(23.1 \%)$ & \\
$2 / 13(15.4 \%)$ & \\
$13 / 13(100 \%)$ & \\
\hline
\end{tabular}

nodules throughout the follow-up, independently of age and sex. Thyroid volume at diagnosis also independently predicted the risk of developing new nodules.

\section{Discussion}

The findings of the present study, performed in a large cohort of patients with CAT followed by US for a median time of 10.0 years, can be summarized as follows: (1) The appearance of new thyroid nodules is not a rare event being found in $14.7 \%$ patients with CAT; (2) new nodules appeared at significantly higher rate in CAT patients with thyroid nodules at diagnosis compared with those having a nodule-free CAT ( $29.5 \%$ vs $10.3 \%$, respectively); (3) all newly appeared thyroid nodules were benign at FNAC analysis; and (4) multivariate analysis identified thyroid volume and presence or absence of nodule/s at diagnosis as significantly related covariates for newnodule development.

The main result of our study was that, during a 10-year follow-up observation, CAT did not represent a risk factor for the development of clinically overt DTC. This finding argues against the hypothesis that CAT is pathogenetically involved in the development of DTC. Rather, new thyroid nodules more frequently appeared in patients having a larger thyroid gland and thyroid nodules at diagnosis. This observation suggests that environmental and genetic factors involved in the development of nodular goiter play a major role in the appearance of new nodules even in patients with CAT $(31,32)$. The present study was performed in an area of mild iodine deficiency and it should be remembered that iodine intake status, which was not assessed in our series of patients, is indeed a major factor driving development of thyroid nodularity as reported by epidemiological studies (33). Previous studies highlighted the role of higher serum levels of TSH as a risk factor for DTC development (34) being nodular autonomy a protective factor $(35,36)$. Furthermore, it was reported that Tg $\mathrm{Ab}$ but not TPO Ab would be associated with increased frequency of DTC $(9,37)$. However, since, in the present study, no case of DTC was observed throughout the follow-up, neither the role of serum TSH levels nor that of specific thyroid circulating antibody could be addressed. It should be noted that the limited number of nodules eligible for FNAB in our series could represent a limitation of this study.

The results of the present study are in agreement with previous studies. In 1985, Holm et al. (38) evaluated the incidence of DTC in 829 patients with CAT and in 829 age- and sex-matched patients with colloid goiter who

Table 3 Logistic regression analysis for assessing the risk of new nodule development.

\begin{tabular}{|c|c|c|c|c|}
\hline & \multirow[b]{2}{*}{$P$ value } & \multirow[b]{2}{*}{$\operatorname{Exp}(B)$} & \multicolumn{2}{|c|}{$95 \% \mathrm{Cl}$ fo $\operatorname{Exp}(\mathrm{B})$} \\
\hline & & & Lower & Upper \\
\hline Age (years) & 0.532 & 0.994 & 0.975 & 1.013 \\
\hline Gender (M/F) & 0.674 & 0.797 & 0.276 & 2.298 \\
\hline Thyroid volume (mL) & 0.028 & 1.030 & 1.003 & 1.057 \\
\hline Group (CATNOD-/CATNOD+) & $<0.001$ & 3.337 & 1.942 & 5.734 \\
\hline
\end{tabular}


enlisted in the Swedish cancer register from 1959 to 1981. The incidence of DTC was similar in patients with CAT compared with those having a colloid goiter (two cases in each group). The main limitation of this early study is that no ultrasound evaluation was available; thus, small non-palpable thyroid cancers could have been missed. In a more recent multicenter retrospective study, Radetti et al. (22) assessed the role of CAT on the development of thyroid nodules and DTC in a pediatric population of 904 children and adolescents. During a median (range) ultrasound follow-up of $4.5(1.2-12.8)$ years, 10 DTC cases were recorded making an overall rate of $0.87 \%$. However, DTC was detected in three patients at CAT diagnosis and in five patients by submitting to FNAC nodules which were already present at CAT presentation. In two patients only there was enough evidence for the development of DTC during the follow-up, thus making a $0.2 \%$ rate. Based on our and previous data, it appears that, when evaluating the risk for DTC in patients with CAT, a great discrepancy exists between cross-sectional, mainly surgical, studies, which consistently report an association, and longitudinal investigations, which fail to support this relationship. Thus, it is conceivable to believe that routine thyroid ultrasound examination of CAT patients is responsible for the high prevalence of DTC in cross sectional studies.

Taken together, the reported data support the hypothesis that patients with CAT are not characterized by an increased occurrence of clinically overt DTC, even in the long-term. Consistent with this statement is the study by Dvorkin et al. (15) who reported that, at diagnosis, CAT-associated DTCs are small in size and have a low rate of nodal involvement. These peculiarities suggest an earlier diagnosis and indirectly support the concept that CAT patients represent a more ultrasound screened population (15).

In conclusion, although histology remains the gold standard for diagnosing DTC, based on the results of the present study, CAT appears to be associated with a negligible risk of developing clinically overt DTC.

\section{Declaration of interest}

The authors declare that there is no conflict of interest that could be perceived as prejudicing the impartiality of the research reported.

\section{Funding}

This work did not receive any specific grant from any funding agency in the public, commercial, or not-for-profit sector.

\section{References}

1 Dailey ME, Lindsay S \& Skahen R. Relation of thyroid neoplasms to Hashimoto disease of the thyroid gland. A.M.A. Archives of Surgery 195570 291-297. (https://doi.org/10.1001/ archsurg.1955.01270080137023)

2 Guarino V, Castellone MD, Avilla E \& Melillo RM. Thyroid cancer and inflammation. Molecular \& Cellular Endocrinology 2010321 94-102. (https://doi.org/10.1016/j.mce.2009.10.003)

3 French JD, Weber ZJ, Fretwell DL, Said S, Klopper JP \& Haugen BR. Tumor-associated lymphocytes and increased FoxP3+ regulatory $\mathrm{T}$ cell frequency correlate with more aggressive papillary thyroid cancer. Journal of Clinical Endocrinology \& Metabolism 201095 2325-2333. (https://doi.org/10.1210/jc.2009-2564)

4 Mantovani A, Allavena P, Sica A \& Balkwill F. Cancer-related inflammation. Nature $2008 \mathbf{4 5 4} 436-444$. (https://doi.org/10.1038/ nature07205)

5 Liotti F, Visciano C \& Melillo RM. Inflammation in thyroid oncogenesis. American Journal of Cancer Research 20122 286-297.

6 Slijepcevic N, Zivaljevic V, Marinkovic J, Sipetic S, Diklic A \& Paunovic I. Retrospective evaluation of the incidental finding of 403 papillary thyroid microcarcinomas in 2466 patients undergoing thyroid surgery for presumed benign thyroid disease. BMC Cancer 201515 330. (https://doi.org/10.1186/s12885-015-1352-4)

7 Azizi G, Keller JM, Lewis M, Piper K, Puett D, Rivenbark KM \& Malchoff CD. Association of Hashimoto's thyroiditis with thyroid cancer. Endocrine-Related Cancer 201421 845-852. (https://doi. org/10.1530/ERC-14-0258)

8 Boi F, Minerba L, Lai ML, Marziani B, Figus B, Spanu F, Borghero A \& Mariotti S. Both thyroid autoimmunity and increased serum TSH are independent risk factors for malignancy in patients with thyroid nodules. Journal of Endocrinological Investigation 201336 313-320. (https://doi.org/10.3275/8579)

9 Kim ES, Lim DJ, Baek KH, Lee JM, Kim MK, Kwon HS, Song KH, Kang MI, Cha BY, Lee KW, et al. Thyroglobulin antibody is associated with increased cancer risk in thyroid nodules. Thyroid 201020 885-891. (https://doi.org/10.1089/thy.2009.0384)

10 Iliadou PK, Effraimidis G, Konstantinos M, Grigorios P, Mitsakis P, Patakiouta F \& Pazaitou-Panayiotou K. Chronic lymphocytic thyroiditis is associated with invasive characteristics of differentiated thyroid carcinoma in children and adolescents. European Journal of Endocrinology 2015173 827-833. (https://doi.org/10.1530/EJE-141046)

11 Paparodis R, Imam S, Todorova-Koteva K, Staii A \& Jaume JC. Hashimoto's thyroiditis pathology and risk for thyroid cancer. Thyroid 201424 1107-1114. (https://doi.org/10.1089/ thy.2013.0588)

12 Repplinger D, Bargren A, Zhang YW, Adler JT, Haymart M \& Chen H. Is Hashimoto's thyroiditis a risk factor for papillary thyroid cancer? Journal of Surgical Research 2008150 49-52. (https://doi. org/10.1016/j.jss.2007.09.020)

13 Mukasa K, Noh JY, Kunii Y, Matsumoto M, Sato S, Yasuda S, Suzuki M, Ito K \& Ito K. Prevalence of malignant tumors and adenomatous lesions detected by ultrasonographic screening in patients with autoimmune thyroid diseases. Thyroid 201121 37-41. (https://doi.org/10.1089/thy.2010.0050)

14 Zhang Y, Dai J, Wu T, Yang N \& Yin Z. The study of the coexistence of Hashimoto's thyroiditis with papillary thyroid carcinoma. Journal of Cancer Research \& Clinical Oncology 2014140 1021-1026. (https:// doi.org/10.1007/s00432-014-1629-z)

15 Dvorkin S, Robenshtok E, Hirsch D, Strenov Y, Shimon I \& Benbassat CA. Differentiated thyroid cancer is associated with less aggressive disease and better outcome in patients with coexisting Hashimotos thyroiditis. Journal of Clinical Endocrinology \& Metabolism 201398 2409-2414. (https://doi.org/10.1210/jc.2013-1309) 
16 Baser H, Ozdemir D, Cuhaci N, Aydin C, Ersoy R, Kilicarslan A \& Cakir B. Hashimoto's thyroiditis does not affect ultrasonographical, cytological, and histopathological features in patients with papillary thyroid carcinoma. Endocrine Pathology 201526 356-364. (https:// doi.org/10.1007/s12022-015-9401-8)

17 Castagna MG, Belardini V, Memmo S, Maino F, Di Santo A, Toti P, Carli AF, Caruso G \& Pacini F. Nodules in autoimmune thyroiditis are associated with increased risk of thyroid cancer in surgical series but not in cytological series: evidence for selection bias. Journal of Clinical Endocrinology \& Metabolism 201499 3193-3198. (https://doi. org/10.1210/jc.2014-1302)

18 Donangelo I, Walts AE, Bresee C \& Braunstein GD. Lymphocytic thyroiditis is associated WITH increased number of benign cervical nodes and fewer central neck compartment metastatic lymph nodes in patients with differentiated thyroid cancer. Endocrine Practice 2016 22 1192-1198. (https://doi.org/10.4158/E151078.OR)

19 Giagourta I, Evangelopoulou C, Papaioannou G, Kassi G, Zapanti E, Prokopiou M, Papapostolou K \& Karga H. Autoimmune thyroiditis in benign and malignant thyroid nodules: 16-year results. Head \& Neck 201436 531-535. (https://doi.org/10.1002/hed.23331)

20 Kim SK, Woo JW, Lee JH, Park I, Choe JH, Kim JH \& Kim JS. Chronic lymphocytic thyroiditis and BRAF V600E in papillary thyroid carcinoma. Endocrine-Related Cancer 201623 27-34. (https://doi. org/10.1530/ERC-15-0408)

21 Qu N, Zhang L, Lin DZ, Ji QH, Zhu YX \& Wang Y. The impact of coexistent Hashimoto's thyroiditis on lymph node metastasis and prognosis in papillary thyroid microcarcinoma. Tumour Biology 2016 37 7685-7692. (https://doi.org/10.1007/s13277-015-4534-4)

22 Radetti G, Loche S, D'Antonio V, Salerno M, Guzzetti C, Aversa T, Cassio A, Cappa M, Gastaldi R, Deluca F, et al. Influence of Hashimoto thyroiditis on the development of thyroid nodules and cancer in children and adolescents. Journal of the Endocrine Society 20193 607-616. (https://doi.org/10.1210/js.2018-00287)

23 Jackson D, Handelsman RS, Farrá JC \& Lew JI. Increased incidental thyroid cancer in patients With subclinical chronic lymphocytic thyroiditis. Journal of Surgical Research 2020245 115-118. (https:// doi.org/10.1016/j.jss.2019.07.025)

24 Jankovic B, Le KT \& Hershman JM. Clinical review: Hashimoto's thyroiditis and papillary thyroid carcinoma: is there a correlation? Journal of Clinical Endocrinology \& Metabolism 201398 474-482. (https://doi.org/10.1210/jc.2012-2978)

25 Haymart MR, Banerjee M, Reyes-Gastelum D, Caoili E \& Norton EC. Thyroid ultrasound and the increase in diagnosis of low-risk thyroid cancer. Journal of Clinical Endocrinology \& Metabolism 2019104 785-792. (https://doi.org/10.1210/jc.2018-01933)

26 Dal Maso L, Panato C, Franceschi S, Serraino D, Buzzoni C, Busco S, Ferretti S, Torrisi A, Falcini F, Zorzi M, et al. The impact of overdiagnosis on thyroid cancer epidemic in Italy, 1998-2012. European Journal of Cancer 201894 6-15. (https://doi.org/10.1016/j.ejca.2018.01.083)

27 Rotondi M, Coperchini F, Magri F \& Chiovato L. Serum-negative autoimmune thyroiditis: what's in a name? Journal of Endocrinological
Investigation 201437 589-591. (https://doi.org/10.1007/s40618-0140083-8)

28 Croce L, De Martinis L, Pinto S, Coperchini F, Dito G, Bendotti G, Pasquali D, Cappelli C, Latrofa F, Magri F, et al. Compared with classic Hashimoto's thyroiditis, chronic autoimmune serum-negative thyroiditis requires a lower substitution dose of L-thyroxine to correct hypothyroidism. Journal of Endocrinological Investigation 2020 [Online ahead of print]. (https://doi.org/10.1007/s40618-02001249-x)

29 Russ G, Bonnema SJ, Erdogan MF, Durante C, Ngu R \& Leenhardt L. European thyroid association guidelines for ultrasound malignancy risk stratification of thyroid nodules in adults: the EU-TIRADS. European Thyroid Journal 20176 225-237. (https://doi. org/10.1159/000478927)

30 Cibas ES \& Ali SZ. The Bethesda system for reporting thyroid cytopathology. Thyroid 200919 1159-1165. (https://doi.org/10.1089/ thy.2009.0274)

31 Hegedüs L. Clinical practice. The thyroid nodule. New England Journal of Medicine 2004351 1764-1771. (https://doi.org/10.1056/ NEJMcp031436)

32 Durante C, Costante G, Lucisano G, Bruno R, Meringolo D, Paciaroni A, Puxeddu E, Torlontano M, Tumino S, Attard M, et al. The natural history of benign thyroid nodules. JAMA 2015313 926-935. (https://doi.org/10.1001/jama.2015.0956)

33 Aghini-Lombardi F, Antonangeli L, Martino E, Vitti P, Maccherini D, Leoli F, Rago T, Grasso L, Valeriano R, Balestrieri A, et al. The spectrum of thyroid disorders in an iodine-deficient community: the Pescopagano survey. Journal of Clinical Endocrinology \& Metabolism 199984 561-566. (https://doi.org/10.1210/jcem.84.2.5508)

34 Fiore E, Latrofa F \& Vitti P. Iodine, thyroid autoimmunity and cancer. European Thyroid Journal 20154 26-35. (https://doi. org/10.1159/000371741)

35 Fiore E, Rago T, Provenzale MA, Scutari M, Ugolini C, Basolo F, Di Coscio G, Berti P, Grasso L, Elisei R, et al. Lower levels of TSH are associated with a lower risk of papillary thyroid cancer in patients with thyroid nodular disease: thyroid autonomy may play a protective role. Endocrine-Related Cancer 200916 1251-1260. (https:// doi.org/10.1677/ERC-09-0036)

36 Haymart MR, Repplinger DJ, Leverson GE, Elson DF, Sippel RS, Jaume JC \& Chen H. Higher serum thyroid stimulating hormone level in thyroid nodule patients is associated with greater risks of differentiated thyroid cancer and advanced tumor stage. Journal of Clinical Endocrinology \& Metabolism 200893 809-814. (https://doi. org/10.1210/jc.2007-2215)

37 Azizi G \& Malchoff CD. Autoimmune thyroid disease: a risk factor for thyroid cancer. Endocrine Practice 201117 201-209. (https://doi. org/10.4158/EP10123.OR)

38 Holm LE, Blomgren $\mathrm{H} \&$ Löwhagen T. Cancer risks in patients with chronic lymphocytic thyroiditis. New England Journal of Medicine 1985312 601-604. (https://doi.org/10.1056/ NEJM198503073121001)

Received 16 April 2020

Revised version received 17 June 2020

Accepted 22 June 2020 